solcher Therapieversuch gerechtfertigt sein, sagte der Dermatologe.

Eine intraorale PDT könnte auch neue Perspektiven beim oralen Lichen planus eröffnen. In einer aktuellen Studie wurden bei 30 Patienten die Effekte einer Mundspülung mit Dexamethason (vier- mal täglich über zwei Wochen) und einer intraoralen PDT mit dem Photosensibilisator Methylenblau (vier Anwendungen in zwei Wochen) verglichen [Bakhtiari S et al. Photodiagnosis Photodyn Ther 2017; 20: 159-64]. Beide Therapiestrategien führten $\mathrm{zu}$ einem signifikanten Rückgang der Symptome und waren in ihrer Effektivität vergleichbar. Angelika Bauer-Delto

Vortrag „Hot Topic: Mundschleimhauterkrankungen", Thomas Dirschka (Wuppertal), Wiesbaden, 11.11.2017

\section{Risiko für hellen Hautkrebs steigt mit der Körpergröße}

$\mathrm{V}$ on verschiedenen persönlichen Eigenschaften ist bekannt, dass sie das individuelle Risiko für hellen Hautkrebs erhöhen. Dazu zählen ein heller Hauttyp und damit verbunden die Bräunungsfähigkeit der Haut sowie die Haarfarbe.

Auch die Körpergröße als Erwachsener hat Einfluss auf die Wahrscheinlichkeit, an Hautkrebs zu erkranken. Die Assoziation zwischen einer größeren Körperlänge und einem erhöhten Risiko für das Melanom wurde bereits in früheren Untersuchungen aufgezeigt, be- richtete Prof. Günther Hofbauer, Zürich. Eine prospektive US-amerikanische Untersuchung ging jetzt der Frage nach, ob die Körpergröße als Erwachsener auch mit dem Risiko für nicht melanozytären Hautkrebs in Beziehung steht. Basis waren die Daten der Nurses' Health Study mit 117.863 Personen sowie der Health Professionals Follow-up Study mit 51.111 Personen. Darüber hinaus wurden genetische Datensätze dieser Studien genutzt, um die Relation zwischen Körpergröße-assoziierten ge- netischen Markern und dem Risiko für Basalzellkarzinome (3.989 Fälle und 8.530 Kontrollen) sowie für Plattenepithelkarzinome (527 Fälle und 8.962 Kontrollen) zu überprüfen.

Nach Ausschluss von verzerrenden Faktoren ergab sich pro $10 \mathrm{~cm}$ Körpergröße eine Risikoerhöhungen von $9 \%$ für das Plattenepithelkarzinom und von $10 \%$ für das Basalzellkarzinom. Bei einer großen Anzahl von Polymorphismen fand sich keine genetische Assoziation [Li X et al. Br J Cancer 2017; 116: 134-40]. Angelika Bauer-Delto

Vortrag "Non-Melanoma Skin Cancer", Günther Hofbauer (Zürich), Wiesbaden, 11.11.2017

\section{Digitale Hautkrebserkennung - bald Realität?}

$\mathrm{D}$ ie Diagnose von malignen Melanomen erscheint dem erfahrenen Dermatologen in den meisten Fällen unproblematisch. Dennoch hat sich gezeigt, dass durch eine Blickdiagnostik allein nur etwa $70 \%$ aller Melanome korrekt erkannt werden, sagte Prof. Axel Hauschild, Kiel. Die Auflichtmikroskopie und auch die Videoauflichtmikroskopie können die Detektionsrate auf maximal $90 \%$ erhöhen. Bei Nicht-Dermatologen liege die Rate korrekter Diagnosestellungen noch deutlich niedriger.

Als „Paukenschlag, den jeder Dermatologe kennen sollte“, bezeichnete der Hautkrebsexperte eine aktuelle Publikation [Esteva A et al. Nature 2017; 542: 115-18], in der ein künstliches neuronales Netzwerk („deep convolutional neural network“, CNN) zur digitalen Hautkrebserkennung vorgestellt wird: Das lernfähige Bildauswertungsprogramm wurde zunächst mit 1,28 Millionen Bildern auf die Unterscheidung unter- schiedlichster Objekte wie Autos, Tiere oder Häuser und anschließend mit 129.450 klinischen Bildern zu mehr als 2.000 verschiedenen Dermatosen trainiert. Anhand eines Bilder-Sets von Biopsie-gesicherten benignen und malignen Hauttumoren wurden schließlich die Fähigkeiten des CNN im Vergleich $\mathrm{zu}$ denen trainierter Dermatologen überprüft, Karzinome von benignen seborrhoischen Keratosen und Melanome von benignen Nävi zu unterscheiden. Das CNN wies ein extrem gutes Verhältnis von Sensitivität zu Spezifität auf und damit zwischen richtigen Diagnosestellungen und falsch-positiven Befunden (AUC unter der ROC-Kurve: für die Erkennung von Karzinomen anhand klinischer Bilder 0,96, für die Erkennung von Melanomen anhand klinischer Bilder 0,94 bzw. anhand dermatoskopischer Bilder 0,91). Und: Nur einer von 22 Dermatologen war der künstlichen Intelligenz hinsichtlich der Melanom-Er- kennung überlegen und keiner der 25 angetretenen Dermatologen hinsichtlich der Erkennung von Karzinomen.

Ein solches neuronales Netzwerk soll nun als App auf den Markt gebracht werden und könnte künftig von mehr als 6 Milliarden Smartphone-Nutzern verwendet werden. Ein großer Teil von diesen hat keinen so guten Zugang zum Hautkrebsscreening beim Dermatologen wie Patienten in Deutschland, so Hauschild. Das Tool könnte daher die Eigenuntersuchung unterstützen und bei einem verdächtigen Befund motivieren, einen Hautarzt aufzusuchen. Die Sensitivität der digitalen Hautkrebserkennung liegt jedoch nicht bei $100 \%$ falsch-negative Ergebnisse sind daher möglich und könnten den Betroffenen in trügerischer Sicherheit wiegen. Als zusätzliche Diagnostikmethode aber habe das digitale Bildauswertungsprogramm ein großes Zukunftspotenzial, so Hauschild. Angelika Bauer-Delto

Vortrag „Melanom“, Axel Hausschild (Kiel), Wiesbaden, 11.11.2017 